\title{
O DIAGNÓSTICO DE ENFERMAGEM NO CONTEXTO DAS AÇÕES DE ENFERMAGEM: PERCEPÇÃO DOS ENFERMEIROS DOCENTES E ASSISTENCIAIS
}

\author{
THE NURSING DIAGNOSIS OF THE NURSING ACTIONS: PERCEPTION OF THE GRADUATE NURSING AND \\ NURSING CARE
}
EL DIAGNOSTICO DE ENFERMERÍA DE LAS ACIONES DE ENFERMERÍA: PERCEPCIOÓN DE LOS ENFERMEROS MAESTROS E ASISTENCIAIS

\section{Franciele Foschiera ${ }^{1}$ Cláudia Silveira Viera ${ }^{2}$}

\begin{abstract}
RESUMO: O objetivo deste estudo foi verificar qual o conhecimento dos enfermeiros acerca do diagnóstico de enfermagem e perceber se há ou não aplicabilidade do diagnóstico de enfermagem no planejamento da assistência. A metodologia utilizada foi a pesquisa qualitativa junto a enfermeiros atuantes em dois hospitais e docentes de um curso de graduação em Enfermagem da região Oeste do Paraná. Os resultados indicaram que o diagnóstico não é utilizado devido a falta de recursos humanos nas instituições hospitalares pesquisadas. Concluímos que apesar dos ensinamentos na graduação há dificuldades para a aplicação do diagnóstico de enfermagem na prática profissional. Outro ponto negativo foi a constatação que a documentação dos cuidados de enfermagem é sensivelmente deficiente e a transmissão oral das informações sobre o paciente dificulta o planejamento das ações da equipe de enfermagem.
\end{abstract}

PALAVRAS CHAVE: Diagnóstico de enfermagem; Processo de Enfermagem; Prática Profissional.

ABSTRACT: This study sought to verify the nurse's knowledge about the nursing diagnosis and to perceive if there are or not applicability of it in care planning nursing process. The methodological approach was the Field Research with hospital nurses and professor of a Nursing College in western region of Paraná state. As result was found that, nursing diagnosis isn't used because there's a lack of human resources in these hospitals. Although graduation learning, there are difficulties to nursing diagnosis application by nurses. Another negative point was that nursing care notes is sensibly faulty and the oral transmission of the patient's information difficult nursing care planning.

KEY WORDS: Nursing Diagnosis; Nursing Process, Professional Practice.

RESUMEN: Este estudio intentó verificar el conocimiento de la enfermera sobre diagnostico de enfermería y percibirlo si hay o no aplicabilidad de esto en proceso de planeamiento del cuidado. Para tanto se utilizó la pesquisa de campo como principio metodológico, con las enfermeras hospitalarias y profesores de una Facultad de Enfermería en la región occidental del estado de Paraná. Como resultado fue encontrado que, el diagnostico de enfermería no es mucho utilizado porque hay una carencia profesionales en los hospitales. Aunque es en la graduación que aprende, existe dificultades al uso del diagnostico de enfermería. Otro punto negativo se fue la constatación de que los registros de cuidados de enfermería es sensible culpables y t él transmisión oral de las informaciones a respecto del paciente, dificulta el planeamiento de las acciones del equipo de enfermería.

PALABRAS CLAVE: Diagnostico de Enfermería, Proceso de Enfermería, Practica Profesional.

\footnotetext{
${ }^{1}$ Enfermeira. Prefeitura Municipal de Cascavel (Paraná) - Secretaria Municipal de Saúde. Pós-graduada em Enfermagem com ênfase em centro cirúrgico. smfran@pop.com.br

2 Enfermeira, Mestre em Enfermagem Fundamental. Docente da área de Enfermagem Materno Infantil da UNIOESTE. Doutoranda em Enfermagem pela ERRP-USP. Curso de Enfermagem UNIOESTE. Rua Universitária, 2069; Jardim Universitário, CEP 85819-110, Cascavel, Paraná. CP - 711. victorclaudia@uol.com.br
} 


\section{INTRODUZINDO A TEMÁTICA}

A enfermagem brasileira tal qual a mundial, inicialmente era praticada por freiras que prestavam assistência por caridade aos doentes e miseráveis nas santas casas, portanto, neste período os cuidados prestados aos pacientes eram de cunho religioso, não tendo assim nenhum embasamento científico, mas apenas empírico. A assistência era realizada sem o uso de uma metodologia de trabalho para orientar suas ações, conforme as necessidades surgiam as decisões eram tomadas.

Ao longo dos anos a enfermagem vem se desenvolvendo, e para isto, passando por várias fases desde os tempos das civilizações mais antigas, onde as pessoas que prestavam cuidados aos doentes 0 faziam apenas por caridade. A partir do século XIX Florence Nighingale traz um novo enfoque para a Enfermagem e o seu desenvolvimento científico. No Brasil, Wanda de Aguiar Hora foi um importante marco no sentido de propor uma assistência de enfermagem sistematizada. Porém, desde o início, algumas dificuldades foram encontradas como o desconhecimento dos sintomas, das necessidades básicas alteradas e da nomenclatura destas necessidades, dentre outros motivos.

O processo de enfermagem por ter origem nas práticas da enfermagem, possui fases interdependentes e complementares e quando realizadas concomitantemente resultam em intervenções satisfatórias para o paciente. Estas fases compreendem, 0 histórico, o diagnóstico, o plano assistencial, prescrição, evolução e prognóstico (HORTA, 1979).

Dentre estas etapas a presente pesquisa optou por dedicar-se ao diagnóstico de enfermagem, por termo observado durante a vida acadêmica que até mesmo os profissionais de enfermagem encontram muitas dificuldades em utilizá-lo e também por perceber como é de grande importância sua utilização na assistência para a tomada de decisão. Sendo assim, o diagnóstico, talvez seja uma das etapas mais complexas, causando muitas divergências na sua realização.

Muitos enfermeiros também deixam de fazer o diagnóstico de enfermagem e com isso, passam a fragmentar os cuidados e os problemas do paciente deixando de vê-los como um todo. Muitas vezes prescrevendo cuidados que não tem relação com os problemas encontrados.

É neste sentido que percebemos que quando não realizamos o diagnóstico de enfermagem perdemos muitas informações importantes sobre 0 paciente e neste percurso o enfermeiro acaba esquecendo o significado da coleta de dados que realiza, suas interpretações e avaliação dos resultados e as intervenções acabam sendo falhas.

Enquanto um instrumento de trabalho, o diagnóstico de enfermagem proporciona ao enfermeiro um plano de ação, que o aproxima de seu objeto de trabalho através de ações anteriormente refletidas, embasado nos problemas detectados no paciente e, portanto, a produtividade espelha a sensível melhora no processo de trabalho através da qualidade das ações (CROSSETTI, 1995).

É através desta etapa que se torna possível a conclusão do levantamento de dados envolvendo raciocínio e julgamento e é neste sentido que 0 diagnóstico de enfermagem se se torna imprescindível para descrever a relação de ajuda na prática clínica.

A Associação Norte Americana dos Diagnósticos de Enfermagem (NANDA) desenvolveu um sistema de classificação dos diagnósticos que propõe a universalização dos problemas encontrados nos pacientes pelos enfermeiros e diante das várias definições surgidas na literatura afirmando que o diagnóstico de enfermagem é "um julgamento clínico sobre as respostas do indivíduo, da família ou da comunidade aos problemas de saúde/processos vitais, reais ou potenciais. O diagnóstico de enfermagem proporciona seleção das intervenções de 
Enfermagem, v. 06, n. 02, p.189-198, 2004. Disponível em www.fen.ufg.br

enfermagem visando ao alcança dos resultados pelos quais a enfermeira é responsável" (CARPENITO, 2002, p. 33).

Com esta citação, notamos que o diagnóstico de enfermagem quando usado corretamente torna-se um facilitador das ações de enfermagem, pois indicam quais as intervenções que vem ao encontro das necessidades dos pacientes, utilizando-se da avaliação crítica e tomada de decisão, sempre questionando esta veracidade da visão que se tem do diagnóstico de enfermagem.

Durante a formação acadêmica temos a oportunidade de aprender o que é, como utilizar e qual a importância do diagnóstico de enfermagem, pois não serve apenas de auxílio para as intervenções de enfermagem, mas "o diagnóstico pode clarificar a função do enfermeiro ao determinar seu campo de exercício" (GOMES, 1996). No entanto, com o decorrer das aulas práticas supervisionadas observamos que na maioria das vezes, não se faz uso de todas as etapas do processo de enfermagem para a assistência ao indivíduo sob nossos cuidados.

A diferença entre a teoria e a prática que nos é ensinada e a realidade vivenciada despertou interesse em desenvolver este estudo e a partir da coleta de dados aspiramos observar se o diagnóstico é utilizado pelos enfermeiros ou não e se está facilitando as intervenções dos mesmos.

Cabe lembrar que o diagnóstico de enfermagem também fornece critérios mensuráveis para a avaliação da assistência prestada; dá suporte e direção ao cuidado; facilita a pesquisa e o ensino; delimita as funções independentes de enfermagem; estimula o cliente a participar de seu tratamento e do plano terapêutico; e contribui para a expansão de um corpo de conhecimentos próprios para a enfermagem (JESUS, 1995).

Pensando nestes fatores que interferem ou não na aplicação do diagnóstico de enfermagem na prática profissional é que escolhemos o tema para esta pesquisa procurando observar a realidade dos enfermeiros assistenciais de duas instituições da cidade de Cascavel e uma instituição docente da mesma cidade.

Assim, com base no exposto, este estudo buscará saber qual é a compreensão de diagnóstico de enfermagem pelos enfermeiros assistenciais que atuam na área hospitalar e os da docência do curso de Enfermagem de uma universidade da região Oeste do Paraná. Uma vez que para HORTA (1979), o diagnóstico de enfermagem é o segundo passo do processo de enfermagem e definido como a identificação das necessidades do ser humano que precisa de atendimento e a determinação pela enfermeira do grau de dependência deste atendimento em natureza e extensão.

Dessa forma, este estudo propõe como objetivos verificar qual o conhecimento dos enfermeiros a cerca do diagnóstico de enfermagem como fase do processo de enfermagem e perceber se há ou não a aplicabilidade do diagnóstico de enfermagem no planejamento da assistência em instituições hospitalares de Cascavel e no desenvolvimento das aulas práticas supervisionadas dos acadêmicos dessa universidade. E através da obtenção destes, pretendemos sistematizar $\mathrm{o}$ assunto $\mathrm{e}$ proporcionar de forma sucinta subsídios, para pesquisa e conhecimento, a outros profissionais da área de enfermagem.

\section{PROCEDIMENTOS METODOLÓGICOS}

Para viabilizar o alcance dos objetivos propostos neste estudo, consideramos pertinente o desenvolvimento de um estudo descritivo seguindo a metodologia de pesquisa qualitativa e utilizamos como instrumentos de coleta de dados dois questionários, o primeiro para enfermeiros graduados na universidade em que se realizou a pesquisa e o segundo para os professores que faziam parte do corpo docente do curso de Enfermagem dessa universidade.

A população deste estudo é composta por enfermeiros atuantes em duas instituições hospitalares da cidade de Cascavel, conforme aceitação prévia dessas 
instituições para participarem da pesquisa e docentes do curso de Enfermagem também de acordo com a aceitação prévia para participar da pesquisa, de acordo com a aprovação do comitê de ética.

A abordagem dos entrevistados ocorreu em um contato prévio questionando-se o interesse de participação no estudo, seguido do esclarecimento dos propósitos do trabalho e assinatura do termo de consentimento pósinformação. Pedimos permissão também, para trabalhar com os dados que fossem obtidos através dos questionários, mantendo o respeito das informações e sigilo dos sujeitos, preservando o anonimato destes.

Depois de redigido, o questionário foi testado antes de sua utilização definitiva, aplicando alguns exemplares em pequena população, sempre respeitando à Resolução do Conselho Nacional de Saúde n. 196, de 10/10/96 e n. 251 de 07/08/97.

Após o pré-teste percebemos que o questionário entregue aos enfermeiros assistenciais não precisava de ajustes, sendo assim, estes instrumentos foram considerados parte da amostra desta pesquisa. Já o questionário entregue aos enfermeiros docentes da UNIOESTE necessitou alteração na segunda questão, pois as respostas estavam bastante confusas, portanto não estava operativo.

A análise dos dados propõe novas buscas à partir do material coletado, após esta etapa novas hipóteses podem surgir, confirmadas ou não, podendo levantar outras. E a compreensão de todo contexto pode ser ampliada ultrapassando as informações subjetivas e espontâneas MINAYO (1994).

O processo de análise dos dados foi iniciado através da pré-análise, que segundo MINAYO (1994, p. 26), "consiste na escolha dos documentos a serem analisados; na retomada das hipóteses e dos objetivos iniciais da pesquisa, reformulando-as frente ao material coletado; e na elaboração de indicadores que orientem a interpretação final".

Desta forma, as respostas obtidas através dos questionários foram lidas e analisadas conforme o objetivo e a questão proposta no estudo. Elaborando os indicadores para a posterior codificação.

$\mathrm{Na}$ codificação os fenômenos encontrados eram nomeados, cada linha das falas foi lida e analisada. Os núcleos eram sublinhados e retirados dos dados e após a comparação eram agrupados por similaridade e diferenças formando as categorias, que para MINAYO (1994, p. 210) esta fase é "chamada de exploração do material e compreende a classificação e a agregação dos dados, onde são escolhidas as categorias teóricas ou empíricas que comandarão a especificação dos temas".

Com a densificação das categorias e a saturação dos dados pudemos então passar a próxima etapa que é a união das categorias e por fim buscar, através do referencial teórico, compreender os dados coletados, fazendo articulações entre os dados e os referenciais teóricos da pesquisa. Conforme MINAYO (1994) promovendo relações entre o concreto e o abstrato, o geral e o particular, a teoria e a prática.

A análise dos dados se deu conforme o referencial metodológico proposto (MINAYO, 1994) que coloca a análise de conteúdo é um conjunto de técnicas de análise que visam obter, por procedimentos sistemáticos e objetivos de descrição do conteúdo das mensagens, indicadores que possibilitam uma interpretação crítica dos dados obtidos sejam eles de senso comum ou subjetivos.

Encontramos dificuldades na coleta de dados, pois uma das instituições onde foi realizada, estava em fase de transição e contratação de novos profissionais, devido a este fato houve muita rejeição em responder o questionário e um sensível número de recusa. Neste hospital foram entregues 10 questionários e apenas seis foram respondidos. Bem como os profissionais demoravam em entregarem o questionário respondido.

$\mathrm{Na}$ análise dos dados utilizamos a fala dos enfermeiros entrevistados para ilustrar as categorias, usamos nomes de frutas para caracterizar os enfermeiros entrevistados, desta forma os 18 enfermeiros foram identificados cada um com um nome de fruta 
FOSCHIERA; Franciele; VIERA; Cláudia Silveira - O diagnóstico de enfermagem no contexto das ações de enfermagem: percepção dos enfermeiros docentes e assistenciais. Revista Eletrônica de Enfermagem, v. 06, n. 02, p.189-198, 2004. Disponível em www.fen.ufg.br

diferente do outro, no sentido de preservar o sigilo dos informantes.

\section{RESULTADOS E DISCUSSÃO}

A amostra entrevistada se caracterizou da seguinte forma, o corpo docente do curso de Enfermagem da universidade em que se deu a pesquisa é composto por 29 enfermeiros, desse total 11 (44,4\%) entrevistados compreenderam a amostra a ser estudada devido aos critérios postos na metodologia. Dentre esses 58,3\% do total da amostra, devolveram os questionários respondidos. Obteve-se como tempo médio de atuação no ensino entre oito e nove anos, compreendendo $57,1 \%$ dos docentes. As áreas de atuação dos enfermeiros docentes foram as mais diversas, tais como, enfermagem psiquiátrica e saúde mental, emergência, UTI, centro cirúrgico, clínica médico-cirúrgica, enfermagem fundamental e ainda, nas disciplinas de assistência de enfermagem à criança sadia e enfermagem em pediatria, obstetrícia e ginecologia.

Uma das instituições que fez parte deste estudo possui sete enfermeiros que atuam no período diurno, sendo destes entrevistados apenas cinco enfermeiros conforme os critérios preconizados, compreendendo 71,4 $\%$ dos enfermeiros. Já a outra instituição, possui 12 enfermeiros no período diurno e apenas seis fazem parte da amostra deste estudo, compreendendo $50 \%$ do total de enfermeiros que atuam na instituição neste período.

Dentre os enfermeiros atuantes nas instituições hospitalares também a área de atuação foi bastante variada. Alguns enfermeiros relataram que faziam supervisão em várias alas e a cada três meses fazem rodízio mudando a área de atuação constantemente.

Quanto ao tempo de atuação obteve-se como tempo médio três anos e meio à sete anos, compreendendo $54,5 \%$ dos enfermeiros entrevistados. Quanto ao tempo de formação, os enfermeiros concluíram a graduação em média há seis anos, compreendendo 18,1 \% destes entrevistados.
Buscando compreender a utilização do processo de enfermagem pelos enfermeiros tanto assistenciais como docentes pudemos identificar seis categorias, descritas e discutidas a seguir: 0 processo de enfermagem não é utilizado, utilização de partes do processo de enfermagem, controvérsias no entendimento do processo de enfermagem, utilizando uma teoria de enfermagem para embasar o processo de enfermagem, fazendo uso de instrumentos que se adaptem a disciplina e iniciando a utilização do processo de enfermagem.

Procurando saber qual a compreensão a cerca do diagnóstico de enfermagem foi possível encontrar cinco categorias denominadas da seguinte forma: Identificação das necessidades do paciente, direcionando a assistência, parte integrante do processo de enfermagem, diagnóstico como meio de determinar as dependências e ações de enfermagem, necessitando de conhecimento científico para realizar o diagnóstico de enfermagem.

$\mathrm{Na}$ categoria $\mathrm{O}$ Processo de Enfermagem não é Utilizado surgiram questões relativas ao processo de trabalho do enfermeiro, mais especificamente das condições de trabalho as quais a categoria está exposta.

A realidade dos profissionais integrantes desta pesquisa é bastante precária, pois há poucos enfermeiros assistenciais acarretando na perca da qualidade da assistência prestada, uma vez que os enfermeiros se tornam plantonistas e priorizam as atividades administrativas a serem realizadas.

Outro fator considerável é o conceito que os enfermeiros tem do processo de enfermagem como uma forma de cobrança como é percebido nesta fala "não utilizo todas as fases do processo porque não é rotina nesta instituição", portanto o processo de enfermagem não é tido como método de trabalho da enfermagem e sim como uma rotina e principalmente como forma de cobrar as ações do enfermeiro. 
FOSCHIERA; Franciele; VIERA; Cláudia Silveira - O diagnóstico de enfermagem no contexto das ações de enfermagem: percepção dos enfermeiros docentes e assistenciais. Revista Eletrônica de Enfermagem, v. 06, n. 02, p.189-198, 2004. Disponível em www.fen.ufg.br

A categoria Utilizando Parte do Processo de Enfermagem foi composta pelo depoimento de enfermeiros que afirmam desenvolver apenas parte do processo de enfermagem e para complementar esta resposta coloca que acabam não transcrevendo as etapas do processo de enfermagem que executam.

Segundo o relato de um entrevistado (enfermeiro maçã) "não é possível planejar sem levantar o histórico, fazer o diagnóstico e sem prever a evolução e o prognóstico do paciente", percebemos que o processo de enfermagem é tido como um método para a assistência de enfermagem utilizada para levantar dados, diagnosticar, prescrever e avaliar.

O processo de enfermagem é o processo de trabalho da enfermagem, ou seja, parte integrante do processo de trabalho em saúde que deve ser compreendido no conjunto das práticas sociais e internamente nos seus diferentes momentos. Devendo, apesar de sua fragmentação, estar interrelacionado e constantemente avaliado conforme as alterações ocorridas com o paciente, vindo ao encontro as suas necessidades e suprindo-as para qualificar as ações da equipe de enfermagem (ALMEIDA, 1998).

A divisão do processo de enfermagem em etapas é artificial, pois o processo só pode ser desenvolvido integralmente com suas etapas inter-relacionadas e recorrentes devido as mudanças contínuas que ocorrem com o paciente, sendo que o que assegura a individualização dos cuidados é a interação entre enfermeiro e o cliente, a família e os outro profissionais da equipe de saúde (HORTA, 1979).

Ainda houveram quatro enfermeiros que caracterizaram uma subcategoria a qual denominamos de a "oralidade ainda presente no processo de enfermagem", achamos de importante relevância a fala (enfermeiro laranja) que afirma "as ações são de determinação verbal, registro apenas em relatório de enfermagem". Notamos então, que a comunicação oral das informações a respeito da evolução do paciente e as ações de enfermagem prestadas são freqüentemente transmitidas de forma verbal. "As enfermeiras confiam na comunicação oral entre a equipe de enfermagem para a transmissão dos comunicados sobre o estados, os achados significativos e as ordens de enfermagem (CARPENITTO, 1997)".Sabemos que muitas das informações que são realmente importantes para que se possam planejar as ações de enfermagem de acordo com as necessidades do paciente, deixam de ser transmitidas, pois não há a documentação das mesmas apenas a transmissão oral dificultando o andamento da equipe e o planejamento dos cuidados prestados ao paciente.

Nesta mesma subcategoria um entrevistado (enfermeira maçã) coloca que "não tenho tempo para transcrever as etapas" e novamente tem-se a afirmação que apesar da realização de "partes das etapas do processo de enfermagem" a documentação é tida como algo que não é necessário e não gostam de fazer. Estas enfermeiras utilizamse da idéia de não ter um tempo definido para a documentação como desculpa para não realizar algo que não gostam (CHAPLIN et al, 1996).

Desta maneira, através da dificuldade de realização e da fragmentação, a maioria dos enfermeiros não consegue priorizar os dados do paciente a serem documentados e acabam transcrevendo todos os dados a seu respeito, levando a documentações ineficazes, repetitivas e vazias deixando a cientificidade comprometida, mas tendo a sensação de dever cumprido.

A categoria Controvérsias no Entendimento do Processo de Enfermagem caracterizou-se pela fala de dois entrevistados que inicialmente relataram realizar todas as etapas do processo de enfermagem mais finalizando a fala colocaram que "ainda não utilizamos o processo na íntegra".

O enfermeiro mamão, também coloca que "tem-se a necessidade de conhecer todas as outras etapas para se chegar ao diagnóstico". 
de enfermagem: percepção dos enfermeiros docentes e assistenciais. Revista Eletrônica de Enfermagem, v. 06, n. 02, p.189-198, 2004. Disponível em www.fen.ufg.br

Ambas as expressões deixam confusa a resposta, podendo ser interpretada tanto como se os enfermeiros aplicam, mas que é falho e não está resultando na qualificação da assistência de enfermagem e a segunda interpretação e talvez a mais adequada é que não se aplica o processo de enfermagem e estão procurando justificar essa situação.

\section{Em Utilizando uma Teoria de} Enfermagem para Nortear o Processo de Enfermagem temos a primeira categoria composta pelos enfermeiros docentes. Nessa categoria temos que 0 processo de enfermagem é um método utilizado para sistematizar as práticas de enfermagem que precisa estar sempre embasado em uma teoria para que as várias etapas aconteçam de acordo com a teoria proposta como por ex: se utilizarmos a teoria de Horta (HORTA, 1979), seguiremos os princípios das necessidades humanas básicas afetadas para direcionar todas as fases desde 0 histórico até o prognóstico, buscando como resultado da assistência proporcionar o autocuidado ao paciente. As fases do processo de enfermagem também podem variar de acordo com a teoria adotada.

Fazendo uso de Instrumentos que se Adaptem a Disciplina esta categoria foi composta por sete enfermeiros que de acordo com suas disciplinas relataram utilizar instrumentos que auxiliem na aplicação do processo de enfermagem. Conforme a fala do enfermeiro pitanga "utilizamos a entrevista de ajuda terapêutica de TRAVELBEE (1979), e através das falas identificamos os problemas de enfermagem e classificamos as necessidades humanas básicas afetadas". Porém "os instrumentos de enfermagem são as habilidades, conhecimentos e atitudes para a execução de uma atividade" (HORTA, 1979 , p. 20). Com isto notamos como é importante ter conhecimento destes e aplicálos conforme a necessidade do paciente possibilitando resultados satisfatórios. Percebe-se então, que cada coleta de dados deve ser realizada conforme a realidade de cada clientela atendida, por isso não se pode ter um formulário único para levantar os dados que permearão todo o processo de enfermagem.

A categoria Iniciando a Utilização do Processo de Enfermagem foi composta pela fala de um entrevistado (enfermeiro abacaxi) que relata "os alunos são orientados a utilizar o SOAP (...) e com isso é realizado as anotações de enfermagem". Nesse momento do curso de enfermagem tem o primeiro contado com o processo de enfermagem utilizando apenas a fase da evolução em uma disciplina do primeiro ano do curso

Conforme o entrevistado relata, os alunos iniciam as aulas práticas supervisionadas realizando apenas as anotações da evolução do paciente e das técnicas desenvolvidas durante o período de estágio. Porém, estes alunos não tiveram embasamento teórico para desenvolver todas as etapas do processo de enfermagem.

Como dito anteriormente, o enfermeiro deveria ser preparado para utilizar o processo e o diagnóstico de enfermagem no primeiro ano da graduação através da introdução de uma disciplina específica direcionada ao ensino da metodologia da assistência possibilitando ao aluno o intercâmbio entre teoria e prática ampliando a visão do processo saúde-doença.

A Identificação das Necessidades do Paciente constitui a primeira categoria da segunda questão do instrumento de coleta de dados entregue aos enfermeiros assistenciais. Esta categoria procurou caracterizar as expressões de cinco entrevistados, dentre eles um entrevistado relatou (enfermeiro carambola) "é um meio de detectar os problemas ocorridos com os pacientes possibilitando tratamento e prevenção".

Esta compreensão que alguns entrevistados possuem a cerca do diagnóstico de enfermagem decorre da teoria de HORTA (1979) das necessidades humanas básicas afetadas que considera as como "os entes da enfermagem" e coloca que as necessidades do ser humano estão em estado de equilíbrio dinâmico e quando em desequilíbrio precisam ser resolvidas e neste 
FOSCHIERA; Franciele; VIERA; Cláudia Silveira - O diagnóstico de enfermagem no contexto das ações de enfermagem: percepção dos enfermeiros docentes e assistenciais. Revista Eletrônica de Enfermagem, v. 06, n. 02, p.189-198, 2004. Disponível em www.fen.ufg.br

momento exigem assistência de
enfermagem.
Para a mesma autora o diagnóstico de
enfermagem é o momento onde através dos dados coletados no histórico de enfermagem torna-se possível identificar estas necessidade humanas básicas afetadas do paciente na tentativa de promover ações que possam beneficiá-los.

Direcionando a Assistência de Enfermagem constitui outra categoria referente a essa questão, em que a fala dos nove entrevistados que compuseram esta categoria, sendo eles três enfermeiros assistenciais hospitalares e seis enfermeiros docentes, o diagnóstico de acordo com o enfermeiro goiaba é caracterizado "como um instrumento que direciona a assistência de enfermagem para todas as ações sejam elas imediatas ou não".

O que o diagnóstico é um instrumento do trabalho da enfermeira, onde podemos estabelecer um plano de ação baseada pelas necessidades do paciente na busca da prevenção e restabelecimento da saúde do mesmo aproximando a enfermeira do seu objeto de trabalho. Portanto, qualifica a assistência de enfermagem na medida em que possibilita e desencadeia a assistência de enfermagem (CROSSETTI, 1995).

Para um dos entrevistados (enfermeiro morango) o diagnóstico de enfermagem é "composto de dados clínicos sobre indivíduos, família, etc sobre seu processo saúde-doença/problemas, nos quais 0 enfermeiro se baseará para realizar suas ações e intervir nos resultados".

Esta expressão parece estar de acordo com a definição do diagnóstico de enfermagem na NANDA que tem como proposta, a seguinte definição do diagnóstico de enfermagem: “... julgamento clínico das respostas do indivíduo, da família ou da comunidade aos processos vitais ou aos problemas de saúde atuais ou potenciais, os quais fornecem a base para a seleção das intervenções de enfermagem, para atingir resultados, pelos quais o enfermeiro é responsável (CARPENITTO, 1997)". Assim, o enfermeiro deve estar freqüentemente disposto a modificar suas ações e adaptá-la às necessidades do paciente, porém, isto só é possível através do conhecimento técnico e científico.

Parte Integrante do Processo de Enfermagem esta categoria foi composta por cinco enfermeiros assistenciais hospitalares e quatro enfermeiros docentes, e caracterizada pela fala do enfermeiro kiwi que relata "é o caminho da prestação da assistência, sem ele não tem como realizar plano assistencial ou prescrição de enfermagem".

Nesta categoria o diagnóstico de enfermagem é visto como parte integrante do processo de enfermagem uma vez que o diagnóstico de enfermagem deriva de avaliações do paciente como um ser autônomo que interage com suas particularidades e com o ambiente a todo o momento (CARPENITTO, 1997). Desta forma, o diagnóstico deve ter continuidade e suas ações devem ser sistematizadas, sendo o diagnóstico parte integrante deste ciclo, mas não pode ser retirado deste contexto, pois resultaria numa assistência inadequada.

Podemos então, confirmar a interrelação das fases do processo e percebemos que o processo de enfermagem é separado em etapas "didaticamente", mas na realidade ele é contínuo e através do uso deste método é que tomamos as decisões corretas para cada situação. 


\section{O Diagnóstico de Enfermagem Como} um Meio de Determinar as Dependências e Ações de Enfermagem é uma categoria que surgiu de falas como a do entrevistado (enfermeiro limão) que definiu o diagnóstico de enfermagem como "determinação pela enfermeira do grau de dependência do atendimento em natureza e extensão". Este conceito também compreende a identificação do grau de dependência quanto a natureza podendo ser total ou parcial e quanto a extensão sendo determinado por indicadores e valores como conhecimento, deambulação, motilidade, estado mental, condições do ambiente e condições sócio-econômicas, portanto a prática do enfermeiro ainda tem como referencial este conceito (HORTA, 1979).

Quatro enfermeiros compuseram a categoria Necessitando de Conhecimento Científico para Realizar o Diagnóstico de Enfermagem que foi caracterizada pela fala do enfermeiro nectarina relatando que "o diagnóstico envolve conhecimento científico do enfermeiro, sensibilidade e compromisso".

Todos os cuidados de enfermagem são baseados em princípios científicos que transformados na prática no indivíduo, sendo estas ações organizadas por diversos profissionais desempenhando seu trabalho (LEOPARDI, 1995). Todo este conjunto de trabalhos, objetos, instrumentos e finalidades é que compõe o processo de enfermagem.

Porém faz-se necessário o domínio da fisiologia, anatomia, sociologia dentre outras ciências para embasar todas as ações direcionadas às necessidades do paciente e tornando-as práticas profissionais.

É com a realização do processo de enfermagem que se aperfeiçoa o profissional, surgindo a satisfação, estimulando a inovação e a criatividade na procura de solução para os problemas de cuidados de enfermagem (IYER et al, 1993).

\section{CONSIDERAÇÕES FINAIS}

Este estudo se desenvolveu a partir do interesse em perceber se os profissionais de enfermagem aplicavam o diagnóstico de enfermagem em suas práticas, porém durante o desenvolvimento vimos que há diferença entre a aplicação do diagnóstico na graduação e após, nas práticas de enfermagem em geral.

Fazendo uma relação entre os dados dos enfermeiros assistenciais das instituições hospitalares da amostra deste estudo e os dados dos enfermeiros docentes, notamos que na graduação os conteúdos a respeito do processo de enfermagem são transmitidos aos acadêmicos e estes são treinados e preparados cientificamente para efetuarem a assistência aos pacientes. Porém, devido a alguns fatores após o dia em que recebem o diploma e tornam-se enfermeiros muitos passam a "esquecer" o conteúdo por vezes não aplicando o processo de enfermagem na assistência.

No decorrer do estudo temos os fatores como os recursos humanos que são deficientes nas instituições em que realizamos a coleta de dados, onde o número de enfermeiros é deficiente e acabam tendo que assumir ações administrativas além da assistência ao paciente e sabemos que muitas vezes os enfermeiros que poderiam delegar as ações administrativas para assumir a assistência direta ao paciente não o fazem.

Outro fator relevante é a jornada dupla de trabalho, vários entrevistados trabalham em duas instituições perfazendo uma jornada de trabalho diária de 12 horas, o resultado está na prestação da assistência que fica inadequada e deficiente, pois o cansaço físico e mental refletem-se nas ações.

Os dados nos levaram a certeza de que o processo de enfermagem é método de trabalho de enfermagem podendo compará-lo a agulha de uma costureira, uma vez que, ambos são instrumentos de trabalho e em ambos os casos os profissionais não podem exercer sua profissão na ausência de seus instrumentos. Também é o que os dados desta pesquisa apontaram e a literatura afirma.

Observamos também que a documentação ainda é muito relapsa, deixando de ser transcrito muitas 
Enfermagem, v. 06, n. 02, p.189-198, 2004. Disponível em www.fen.ufg.br

informações importantes a respeito do paciente, sendo elas transmitidas apenas verbalmente.

Não foi possível observar se a realização do diagnóstico de enfermagem está facilitando ou não as ações de enfermagem através dos dados coletados, pois os enfermeiros assistenciais não realizam esta fase do processo, apenas fazem a evolução do paciente para registrar parte de suas ações. O que foi possível perceber é que nas aulas práticas supervisionadas o diagnóstico é desenvolvido de acordo com os dados coletados na primeira fase do processo de enfermagem e com ela todas as ações são planejadas para que os cuidados sejam a contento.

Notamos então, que "pode não facilitar", mas é imprescindível para orientar a assistência de enfermagem e apontar o melhor caminho na busca do restabelecimento da saúde do paciente.

\section{REFERÊNCIAS BIBLIOGRÁFICAS}

ALMEIDA, M. et al. Dor em pacientes com próteses de quadril: diagnóstico de enfermagem. Rev. Gaúcha Enfermagem, v. 19, n. 1, p 33-37, 1998.

CARPENITTO, L. J. Diagnóstico de enfermagem: aplicação à prática clínica. $6^{\mathrm{a}}$ ed., Porto Alegre: Artes Médicas; 1997.

CARPENITTO, L. J. Diagnósticos de enfermagem: aplicação à prática clínica. 8a .ed., Porto Alegre: Artes Médicas, 2002.
CHAPLIN M. J. M.; GUIMARÃES C. R.; SILVA S. R.; SOARES S. R.;SILVA V. L. Refletindo o processo de enfermagem sob a ótica do cotidiano. Rev. Enfermagem UERJ, v. 4, n. 2, p. 220-224, 1996.

CROSSETTI, M. G. O. Algumas reflexões sobre o diagnóstico de enfermagem e os elementos do processo de trabalho. Texto \& Contexto Enfermagem, v. 4, n. 1, p. 150159,1995

GOMES, L. M. S. M. Diagnóstico!... De Enfermagem? Nursing. n. 106, p. 18-19, 1996.

HORTA, W. A. Processo de enfermagem. São Paulo: EPU; 1979.

IYER, P. W. ; TAPTICH, B. J.; BERNICCHILOSEY, D. Processo e diagnóstico em enfermagem. Porto Alegre Artes Médicas; 1993.

JESUS, C. A.C. Evolução histórica do diagnóstico de enfermagem e sua aplicabilidade no planejamento da assistência. Rev. de Saúde do Distrito Federal, v. 6, n. 1 e 2, p. 37-40, 1995.

LEOPARDI $M$. T. O método como objetificação científica na assistência da enfermagem. Texto \& Contexto Enfermagem. v.4, n. 1, p. 9-18, 1995.

MINAYO, M. C. S. O desafio do conhecimento: pesquisa qualitativa em saúde. $3^{a}$ ed. São Paulo - Rio de Janeiro: Hucitec/Abrasco; 1994.

TRAVELBEE, J. Intervenciõn en enfermeria psiquiatrica. 2. ed. Columbia: Carvajal, 1979.

Texto recebido em 28/04/2004.

Publicação aprovada em 31/08/2004 